\title{
A routine method for the quantification of the novel antimycotic drug posaconazole in plasma using liquid chromatography-tandem mass spectrometry
}

\author{
Michael Vogeser ${ }^{1, *}$, Christina Rieger ${ }^{2}$, Helmut \\ Ostermann ${ }^{2}$ and Ute Spöhrer ${ }^{3}$ \\ ${ }^{1}$ Institute of Clinical Chemistry, Hospital of the \\ University of Munich, Munich, Germany \\ 2 Department of Internal Medicine III, Hospital of the \\ University of Munich, Munich, Germany \\ ${ }^{3}$ Department of Pharmacy, Hospital of the \\ University of Munich, Munich, Germany
}

\begin{abstract}
Background: Posaconazole is now widely used for prophylaxis of invasive fungal infections in immunocompromised patients. The pharmacokinetic properties of the drug argue for therapeutic monitoring, but so far described analytical methods have shortcomings with respect to application in a routine setting. The aim of our work was to develop an analytical method suitable for routine use.

Methods: Liquid chromatography-tandem mass spectrometry (LC-MS/MS) was used. For sample preparation, protein precipitation followed by on-line solid phase extraction was used. SCH 56984, a posaconazole related compound provided by the manufacturer of posaconazole, was used as internal standard.

Results: The method was characterized by short hands-on time and an analytical run time of four minutes. Validation data demonstrated acceptable analytical performance.

Conclusions: LC-MS/MS with on-line solid phase extraction for sample preparation allows the implementation of a convenient and reliable method for pharmacokinetic monitoring of posaconazole.

Clin Chem Lab Med 2009;47;579-84.
\end{abstract}

Keywords: antimycotic drugs; fungal infections; liquid chromatography-tandem mass spectrometry (LC-MS/ MS); posaconazole.

\section{Introduction}

Invasive fungal infections, most frequently caused by Candida spp. and Aspergillus spp. are a leading cause of mortality in severely immunocompromised hemato-oncological patients (1). Therefore, prophylaxis of such infections has a very high priority in the care of

*Corresponding author: Michael Vogeser, MD, Institute of Clinical Chemistry, Hospital of the University of Munich, Marchioninistr. 15, 81377 Munich, Germany Phone: + 49897095 3221, Fax: + 49897095 6220,

E-mail: Michael.Vogeser@med.uni-muenchen.de Received June 24, 2008; accepted February 17, 2009; previously published online March 23, 2009 these patients (2). Itraconazole is widely used for this purpose in neutropenic hematological patients; its use, however, is hampered by rather poor and variable enteral absorption of the oral formulation and variable hepatic metabolism. Due to these pharmacokinetic characteristics, therapeutic drug monitoring of itraconazole is useful, with target concentration for trough levels above $500 \mu \mathrm{g} / \mathrm{L}$ (3). Posaconazole (SCH 56592 ) is a structural homologue of itraconazole; two chlorine atoms are exchanged by fluorine and one $\mathrm{OH}$-function is added. The drug has recently been introduced for prophylaxis of invasive fungal infections $(4,5)$. In two randomised phase III trials, the therapeutic efficacy of posaconazole in the prevention of fungal infection in patients with acute leukemia and graft-vs.-host-disease has been demonstrated $(6,7)$. Posaconazole is available only as an oral suspension, but its absorption appears to be superior to that of itraconazole. However, absorption is greatly influenced by the dosing regimen that is used; for example, twice vs. four times a day dosing for treatment of invasive fungal infections, three times a day dosing for antifungal prophylaxis, or the effect of fat content of concomitant meals $(8,9)$. Typical steady state predose concentrations, maximum concentrations and average concentrations of posaconazole are approximately between 200 and $900 \mu \mathrm{g} / \mathrm{L}$ (10). The drug does not undergo metabolism by cytochrome P450 (CYP) enzymes and $<30 \%$ of an administered dose is typically metabolised, usually by glucuronidation. The compound is primarily excreted into bile (5). Thus, the pharmacokinetic characteristics of posaconazole seem more favorable compared to itraconazole (5). However, monitoring of posaconazole concentrations in plasma may be important, particularly in the context of future clinical trials in selected groups of individuals such as pediatric patients (11). The availability of a routine method for the quantification of posaconazole plasma concentrations may be helpful for patients with severely impaired enteral absorption due to graft-vs-host disease, impaired excretory hepatic function or questionable compliance with drug administration requirements.

The technique of liquid chromatography-tandem mass spectrometry (LC-MS/MS) offers very high specificity and sensitivity, with a short turn-around time, limited requirements of sample preparation, and good practicality. This technology has become available in a growing number of tertiary care hospitals worldwide, despite the high instrument cost. The aim of our work was to develop a convenient LC-MS/MS method for the quantification of plasma posaconazole concentrations. We applied automated on-line solid 
phase extraction prior to mass spectrometric analysis since this approach allows for a more robust and simple technique for a variety of analytes.

\section{Materials and methods}

\section{Analytical procedure}

Chemicals Posaconazole and the compound SCH 56984, a homology of posaconazole, were used as internal standard (Figure $1 \mathrm{~A}$ and $\mathrm{B}$ ). These were obtained from the ScheringPlough Research Institute (Kenilworth, NJ, USA). The lots of posaconazole and SCH 56984 were analysed by ScheringPlough, and XenoBiotic Laboratories Inc., respectively (Plainsboro, NJ, USA). These laboratories certified that the purity was $>98 \%$. Stock solutions of these compounds were made in methanol $(50 \mathrm{mg} / \mathrm{L})$. Methanol and water were of HPLC-grade (Baker, Deventer, The Netherlands); formic acid was from Merck (Darmstadt, Germany).

Instruments A Waters Alliance 2795 HPLC module (Waters, Milford, USA) was used, coupled with a split of approximately 1:10 to a Micromass Quattro LC tandem mass spectrometry system (Waters, Manchester, UK).

\section{Mass spectrometric conditions}

Electrospray ionization in the positive mode was used. Source parameters were tuned to obtain the protonated quasi-molecular ions $\left(\left[\mathrm{M}+\mathrm{H}^{+}\right]\right.$) of posaconazole $(701.4 \mathrm{~m} / \mathrm{z})$ and of the internal standard compound SCH 56984 (687.3 $\mathrm{m} / \mathrm{z}$ ), respectively. The following settings resulted in optimal ion yield: capillary voltage, $3.5 \mathrm{kV}$; cone voltage, $57 \mathrm{~V}$; source temperature, $90^{\circ} \mathrm{C}$; desolvation temperature, $280^{\circ} \mathrm{C}$; nitrogen flow, $\sim 800 \mathrm{~L} / \mathrm{h}$; cone gas flow, $\sim 75 \mathrm{~L} / \mathrm{h}$. The collision energy with argon as the collision gas was $32 \mathrm{~V}$. With these conditions, several intense product ions were generated for both analytes (Figure 2A and $\mathrm{B}$ ). For quantification the following multiple-reaction monitoring (MRM) transitions were recorded: posaconazole 701.4>683.5, SCH 56984 (internal standard) 687.3>669.0. The dwell time for all MRM traces was $0.15 \mathrm{~s}$. Representative chromatograms are given in Figure 3.

\section{Calibration and quality control materials}

A three-point set of lyophilised calibrators from Chromsystems (Munich, Germany) was used together with three levels of lyophilised quality control samples from the same source. Calibrator concentrations were 570, 2280 and $5570 \mu \mathrm{g} / \mathrm{L}$.

\section{Sample preparation and chromatography}

A semi-automated sample preparation protocol with a manual protein precipitation step and automated on-line solid phase extraction employing column-switching was used. Into $1.5 \mathrm{~mL}$ polypropylene cups, $50 \mu \mathrm{L}$ of calibrator or patient sample was precipitated with $200 \mu \mathrm{L}$ of methanol/ acetonitrile (90/10) containing the internal standard compound SCH 56984 at a concentration of $200 \mu \mathrm{g} / \mathrm{L}$. Following vigorous vortex mixing, specimens were centrifuged for $10 \mathrm{~min}$ at $15,000 \mathrm{~g}$ using a non-thermostated standard bench top centrifuge. Next, $100 \mu \mathrm{L}$ of the clear supernatant was transferred into HPLC vials and placed into the HPLC autosampler.

For on-line solid phase extraction, a Waters Oasis HLB ${ }^{\circledR}$ column (25 $\mu \mathrm{m}, 2.1 \times 20 \mathrm{~mm}$; Waters, Milford, USA) was used, together with a six-port high-pressure switching
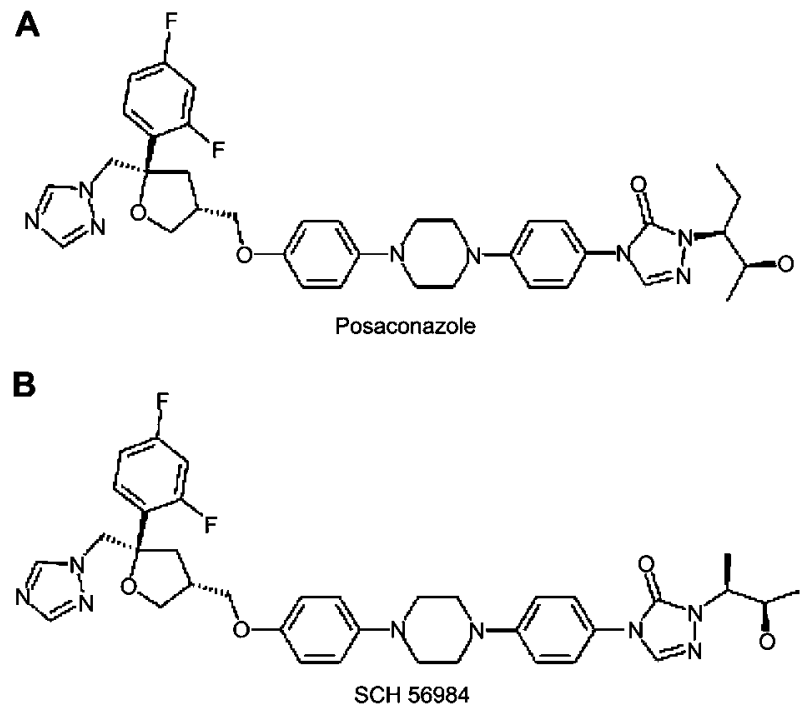

Figure 1 Molecular structure of posaconazole (A) and of the internal standard compound SCH 56984 (B).

\section{A}

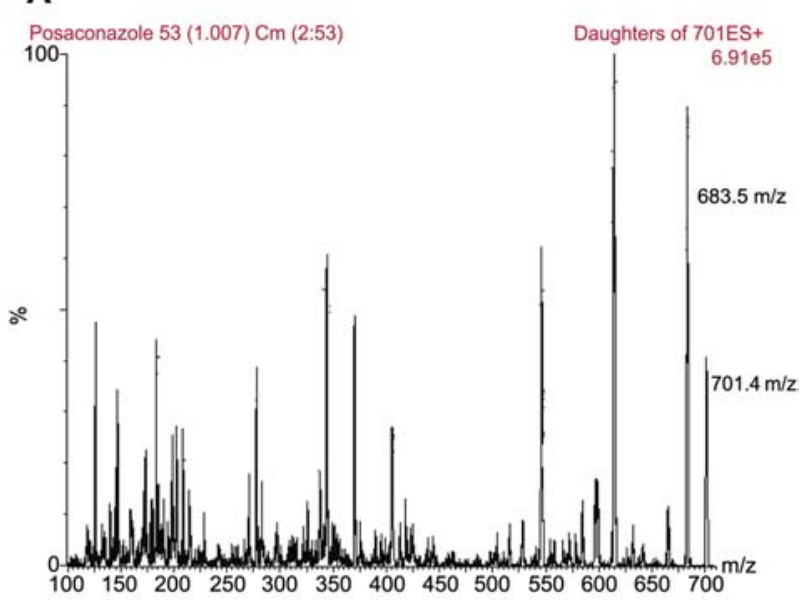

B

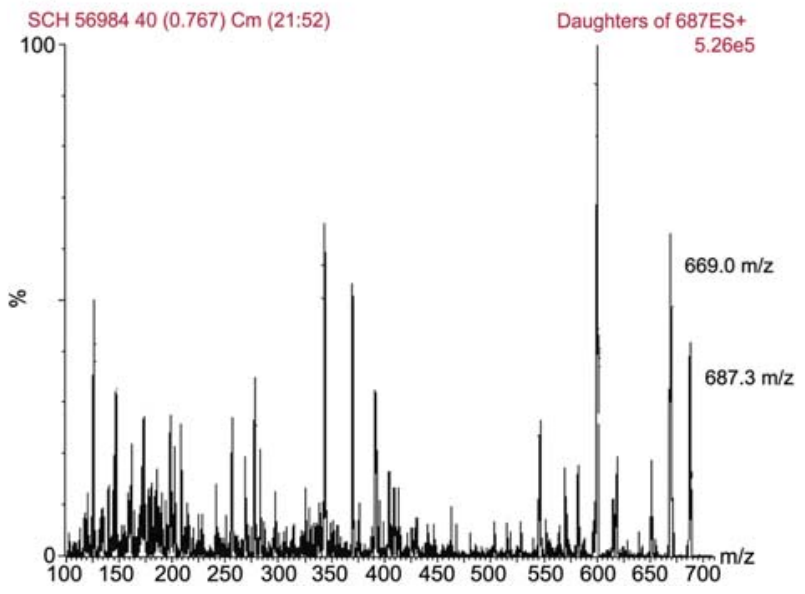

Figure 2 Collision induced disintegration spectrum of posaconazole (A), and internal standard compound SCH 56984 (B) recorded at a collision energy of $29 \mathrm{~V}$.

Posaconazole (not fully disintegrated) precursor ion, 701.4 $\mathrm{m} / \mathrm{z}$; product ion selected for MRM monitoring, $683.5 \mathrm{~m} / \mathrm{z}$. $\mathrm{SCH} 56984$ (not fully disintegrated) precursor ion, 687.3 $\mathrm{m} / \mathrm{z}$; product ion selected for MRM monitoring, $669.0 \mathrm{~m} / \mathrm{z}$. 


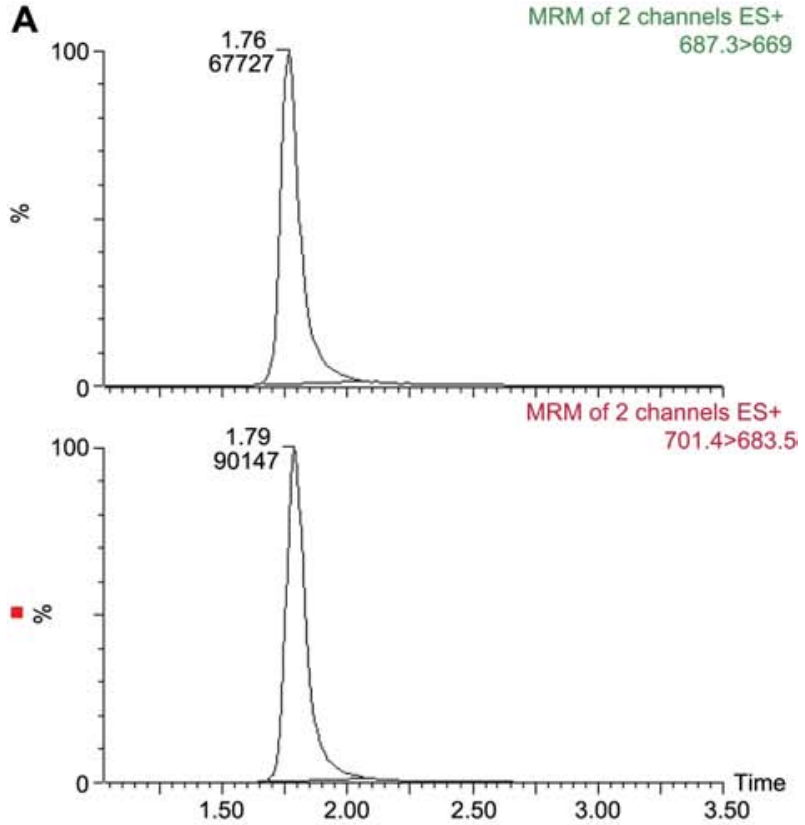

B

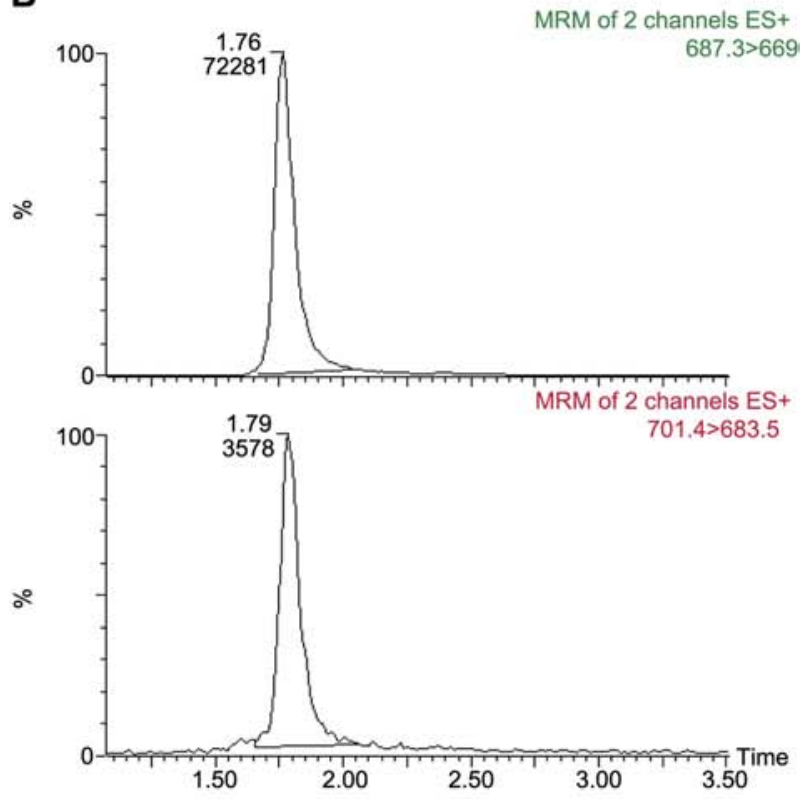

C

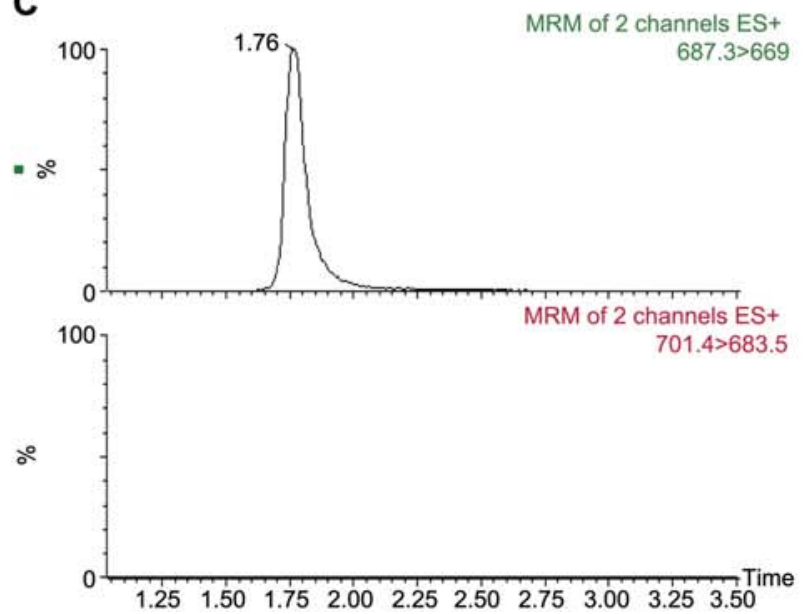

Figure 3 Representative chromatograms.

(A) Patients' plasma (posaconazole concentration $588 \mu \mathrm{g} / \mathrm{L}$ );

(B) plasma sample spiked to a concentration of $30 \mu \mathrm{g} / \mathrm{L}$;

(C) drug-free sample. valve (Rheodyne, Rohnert Park, CA, USA) controlled by the Micromass MassLynx 4.0 mass spectrometry software (Waters, Milford, USA).

The automated extraction procedure consisted of three steps. First $50 \mu \mathrm{L}$ of deproteinized sample was injected and loaded onto the extraction column in valve position A (Figure 4). The mobile phase was water/methanol $90 / 10(v / v)$, delivered at a flow rate of $4 \mathrm{~mL} / \mathrm{min}$. Potentially interfering compounds were washed into the waste. In parallel, the analytical column (LiChrospher ${ }^{\circledR} \mathrm{C} 18$ endcaped $5 \mu \mathrm{m}$, $77 \times 4 \mathrm{~mm}$; Dr. Maisch, Germany) was equilibrated with methanol/0.1\% formic acid $90 / 10(\mathrm{v} / \mathrm{v})$ delivered at a flow rate of $0.9 \mathrm{~mL} / \mathrm{min}$. After $1 \mathrm{~min}$, the switching valve was changed to position $B$; the extraction column was now eluted in a back-flush mode onto the analytical column. After another 2 min, the valve was switched back to position A. During the analytical chromatography into the mass spectrometer in position $A$, the extraction column was washed with acetonitrile/methanol $50 / 50(\mathrm{v} / \mathrm{v})$ at a flow rate of $4 \mathrm{~mL} / \mathrm{min}$ for $1 \mathrm{~min}$ and the extraction column subsequently re-equilibrated with water/methanol 90/10 (v/v). Both the extraction column and analytical column were kept at $38^{\circ} \mathrm{C}$ in the column oven. The retention times of posaconazole and the internal standard SCH 56984 were $\sim 1.8 \mathrm{~min}$ after injection onto the extraction column. The total analytical run-time was $4 \mathrm{~min}$.

For quantification, the peak area-ratios of the MRM trace of posaconazole and the internal standard compound $\mathrm{SCH}$ 56984 were assessed. We did not apply fit weighting or axis transformation.

\section{Validation}

We evaluated the recovery of the two-step extraction process and assessed potential ion-suppression effects. A pure solution of posaconazole $(200 \mu \mathrm{g} / \mathrm{L})$ in water/methanol 90/10 $(\mathrm{v} / \mathrm{v})$ was introduced into the mass spectrometer by direct injection onto the analytical column, in triplicate. The mobile phase consisted of methanol/0.1\% formic acid 90/10 (v/v), and we did not apply on-line solid phase extraction. We used residual clinical samples from 10 intensive care patients not receiving posaconazole to prepare a posaconazole-free plasma pool. We spiked this pool with posaconazole to obtain a concentration of $200 \mu \mathrm{g} / \mathrm{L}$. The spiked pool was submitted in triplicate to the entire analytical process including protein precipitation and automated solid phase extraction. The mean peak areas of the MRM trace of posaconazole for the directly analysed pure solution and for the extracted spiked plasma sample, respectively, were compared.
A

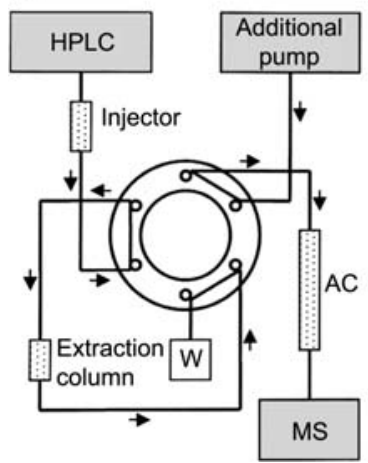

B

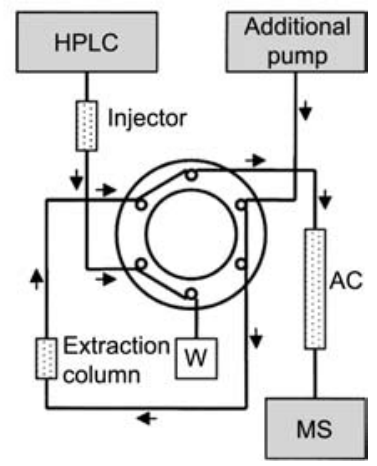

Figure 4 Scheme of the on-line solid phase extraction system.

AC, analytical column; W, waste; MS, mass spectrometry system. 
To verify the specificity of the method, 20 residual plasma samples from patients not treated with posaconazole were analysed. We omitted the addition of internal standard compound to the precipitation solution. MRM chromatograms of posaconazole and the internal standard were inspected for peak signals.

Reproducibility, accuracy and linearity of the method were investigated in three analytical series. Quality control materials at three concentrations and aliquots of a plasma pool from posaconazole-treated patients were quantitated in quadruplicated.

In order to verify the sensitivity of the analytical system at low and sub-therapeutic concentrations, a drug-free plasma was spiked to a concentration of $30 \mu \mathrm{g} / \mathrm{L}$ and the signal to noise ratio was measured for three injections.

Carry over was evaluated by spiking the supernatant of a drug-free plasma sample to a posaconazole concentration of $10,000 \mu \mathrm{g} / \mathrm{L}$ and injected in quadruplicate. The chromatogram of this sample was inspected for peaks in the MRM trace of posaconazole.

The stability of precipitation extracts was evaluated by reanalysis of deproteinized samples of a calibration series, $24 \mathrm{~h}$ after their initial analysis. The peak areas were compared between these two runs.

\section{Results}

The mean peak area for the MRM trace of posaconazole was 803.491 for $200 \mu \mathrm{g} / \mathrm{L}$ posaconazole in water/ methanol, injected without extraction procedure. The mean peak area for a spiked plasma sample containing $200 \mu \mathrm{g} / \mathrm{L}$ posaconazole and submitted to the entire analytical process was 768.649. This corresponds to a signal recovery of $95.7 \%$.

We evaluated 20 plasma samples from patients who were not treated with posaconazole. We observed no peak signals in the MRM traces of the analyte and its internal standard SCH 56984, respectively.

Results for our investigations of imprecision and accuracy are shown in Table 1. The total coefficient of variation was $<6 \%$, and accuracy based on quadruplicate analysis of three quality control samples ranged from $-11.8 \%$ to $11.4 \%$. The CV for the sample pool was $9.7 \%$ and the coefficient for measurement of the calibrators revealed an $r$ value of $>0.99$ for all validation series.

Evaluation of our plasma sample spiked to obtain a posaconazole concentration of $30 \mu \mathrm{g} / \mathrm{L}$ showed a mean signal to noise ratio of $48: 1$. A chromatogram of this sample is shown in Figure 3B.

No carry-over effect was noted. Extracts obtained by protein precipitation were found to be stable for at least $24 \mathrm{~h}$. Chhun et al. (12) has previously reported detailed data on the stability of posaconazole in plasma. We have found our method to be robust after routine use for six months.

\section{Discussion}

We describe a convenient high-throughput LC-MS/MS method for the quantification of posaconazole in human plasma samples. Using commercially available materials for calibration and quality control, validation data demonstrated our method to be compatible with the analytical performance level required for use in the routine clinical laboratory. However, CVs above $5 \%$ and accuracies $> \pm 5 \%$ do not characterize the assay as extraordinarily accurate, as might be expected for a mass spectrometric assay. This observation, however, is typical for a number of LC$\mathrm{MS} / \mathrm{MS}$ assays used in clinical pharmacology. For the majority of these analytes, no stable-isotope labeled internal standard compounds are available and structurally related "homologue" molecules have to be used instead. Often, there is sensitivity drift due to "charging" of the ion source or of the ion optics. This phenomenon seems to affect the target analyte and corresponding internal standard to a different degree, potentially resulting in limitations in accuracy of LCMS/MS methods.

The availability of an appropriate internal standard is critical for the implementation of LC-MS/MS assays for therapeutic drug monitoring. We tried to use fenbuconazole, a commercially available agrochemical antimycotic azole compound, as the internal standard for quantification of posaconazole. Although similar in molecular structure, the ionization behavior of this compound is too different from that of posaconazole to be successfully applied as the internal standard. We observed unacceptable reproducibility ( $C V>15 \%)$ and linearity $(r<0.95)$ when using fenbuconazole as the internal standard for quantification of posaconazole. Development of the present method was only possible after obtaining SCH 56984 from the manufacturer of posaconazole. SCH 56984 is a closely related compound of posaconazole that we used as the internal standard. Unfortunately, this compound is not commercially available at present. Our observation that fenbuconazole was not applicable for use as the internal standard for quantitation of posaconazole might be instrument specific. The development and validation of an LC-MS/MS method using one specific instrument may not be easily transferable to another similar instrument. Even for methods developed for one specific instrument, changes in the performance characteristics due to, for example, contamination of

Table 1 Results of the method validation study.

\begin{tabular}{llll}
\hline & QC level I & QC level II & QC level III \\
\hline Target concentration & $250 \mu \mathrm{g} / \mathrm{L}$ & $1050 \mu \mathrm{g} / \mathrm{L}$ & $4190 \mu \mathrm{g} / \mathrm{L}$ \\
Measured concentration (mean) & $245 \mu \mathrm{g} / \mathrm{L}$ & $1066 \mu \mathrm{g} / \mathrm{L}$ & $4298 \mu \mathrm{g} / \mathrm{L}$ \\
Coefficient of variation & $5.8 \% \mu \mathrm{g} / \mathrm{L}$ & $5.4 \% \mu \mathrm{g} / \mathrm{L}$ & $5.1 \% \mu \mathrm{g} / \mathrm{L}$ \\
Accuracy (mean concentration) & $-2.1 \%$ & $+1.5 \%$ & $+2.6 \%$ \\
Accuracy (range) & $-11.8 \% \pm 3.9 \%$ & $-7.2 \% \pm 8.2 \%$ & $-6.2 \% \pm 11.4 \%$ \\
\hline
\end{tabular}


the ion source or the mass analyzer, or accumulation of late eluting matrix compounds such as phospholipids on the analytical column causing ion suppression, may compromise the validity of the results if no stable isotope-labeled internal standard compound is available. Therefore, strict adherence to good quality assurance practices during development and application of LC-MS/MS methods is critical.

High analytical specificity, simple sample preparation, and high throughput are typical strengths of LC-MS/MS technology used for therapeutic drug monitoring (13). High analytical precision is, however, not a strength of this technology. It is well recognized that individual HPLC-UV methods may be superior to LC-MS/MS methods with respect to precision.

Several HPLC methods for the quantitation of posaconazole in human plasma employing ultraviolet detection have been described previously (14-16). These methods require laborious sample preparation along with extensive chromatographic run times. In addition, a major limitation of conventional HPLC methods that rely on UV detection is the questionable specificity for individual samples. Analytical specificity of HPLC-UV methods can be evaluated for interference from endogenous compounds as well as commonly used compounds that may be co-administered with the drug being measured. However, spurious results caused by co-elution of interfering exogenous compounds with the analyte or internal standard compound is a potential problem with this method of detection. With respect to specificity, LC$\mathrm{MS} / \mathrm{MS}$, although requiring substantially more expensive instrumentation, has emerged as the standard technology for therapeutic drug monitoring and is replacing HPLC-UV methods in a growing number of clinical laboratories.

Three mass spectrometric methods for the quantification of posaconazole in human plasma have been published previously (16-18). Some of these methods are laborious, employing solvent extraction with an evaporation step (18). Others require robotic sample preparation systems $(16,17)$. In terms of practicability, the method we describe is superior to these previous methods since only protein precipitation is needed as the manual sample preparation step. This is next followed by automated solid phase extraction with a permanently used extraction cartridge employing standard chromatography equipment. This technique of on-line solid phase extraction has been applied to a variety of LC-MS/MS methods. We have previous extensive experience with therapeutic drug monitoring of immunosuppressants and itraconazole $(19,20)$. Applying this straightforward principle of sample clean-up, efficient control of ion suppression effects and a very high degree of technical robustness can be realized for high-throughput routine methods. This technology also helps minimize the health hazards associated with exposure to organic solvents used in solvent extraction protocols, and is far more economical compared with single-use solid phase extraction cartridges.

In summary, our results suggest that the semi-automated LC-MS/MS method we describe is applicable for monitoring of posaconazole plasma concentrations in large-scale clinical studies, as well as for routine therapeutic drug monitoring.

\section{Acknowledgements}

We thank the Schering-Plough Research Institute, Kenilworth, USA, and Essex Pharma GmbH, Munich, Germany, for providing us with posaconazole and compound $\mathrm{SCH}$ 56984.

\section{References}

1. Maschmeyer G, Haas A, Cornely OA. Invasive aspergillosis: epidemiology, diagnosis and management in immunocompromised patients. Drugs 2007;67:1567-60‥

2. Ullmann AJ, Cornely OA. Antifungal prophylaxis for invasive mycoses in high risk patients. Curr Opin Infect Dis 2006;19:571-6.

3. Glasmacher A, Hahn C, Leutner C, Molitor E, Wardelmann E, Losem C, et al. Breakthrough invasive fungal infections in neutropenic patients after prophylaxis with itraconazole. Mycoses 1999;42:443-51.

4. Nagappan V, Deresinski S. Reviews of anti-infective agents: posaconazole: a broad-spectrum triazole antifungal agent. Clin Infect Dis 2007;45:1610-7.

5. Schiller DS, Fung HB. Posaconazole: an extended-spectrum triazole antifungal agent. Clin Ther 2007;29:186286.

6. Cornely OA, Maertens J, Winston DJ, Perfect J, Ullmann AJ, Walsh TJ, et al. Posaconazole vs. fluconazole or itraconazole prophylaxis in patients with neutropenia. $\mathrm{N}$ Engl J Med 2007;356:348-59.

7. Ullmann AJ, Lipton JH, Vesole DH, Chandrasekar $P$, Langston A, Tarantolo SR, et al. Posaconazole or fluconazole for prophylaxis in severe graft-versus-host disease. N Engl J Med 2007;356:335-47.

8. Courtney R, Radwanski E, Lim J, Laughlin M. Pharmacokinetics of posaconazole coadministered with antacid in fasting or nonfasting healthy men. Antimicrob Agents Chemother 2004;48:804-8.

9. Courtney R, Wexler D, Radwanski E, Lim J, Laughlin M. Effect of food on the relative bioavailability of two oral formulations of posaconazole in healthy adults. $\overline{\mathrm{Br}} \mathrm{J} \overline{\mathrm{Clin}}$ Pharmacol 2004;57:218-22.

10. Ullmann AJ, Cornely OA, Burchardt A, Hachem R, Kontoyiannis DP, Töpelt $K$, et al. Pharmacokinetics, safety, and efficacy of posaconazole in patients with persistent febrile neutropenia or refractory invasive fungal infection. Antimicrob Agents Chemother 2006;50:658-66.

11. Smith J, Andes D. Therapeutic drug monitoring of antifungals: pharmacokinetic and pharmacodynamic considerations. Ther Drug Monit 2008;30:167-72.

12. Chhun S, Rey E, Tran A, Lortholary O, Pons G, Jullien V. Simultaneous quantification of voriconazole and posaconazole in human plasma by high-performance liquid chromatography with ultra-violet detection. J Chromatogr B Analyt Technol Biomed Life Sci 2007;852:223-8.

13. Vogeser M, Seger C. A decade of HPLC-MS/MS in the routine clinical laboratory - goals for further developments. Clin Biochem 2008;41:649-62.

14. Kim H, Kumari $P$, Laughlin M, Hilbert MJ, Indelicato SR, Lim J, et al. Use of high-performance liquid chromatographic and microbiological analyses for evaluating the presence or absence of active metabolites of the antifungal posaconazole in human plasma. J Chromatogr A 2003;987:243-8.

15. Müller C, Arndt M, Queckenberg C, Cornely OA, Thei- 
sohn M. HPLC analysis of the antifungal agent posaconazole in patients with haematological diseases. Mycoses 2006;49(Suppl 1):17-22.

16. Krieter P, Flannery B, Musick T, Gohdes M, Martinho M, Courtney R. Disposition of posaconazole following single-dose oral administration in healthy subjects. Antimicrob Agents Chemother 2004;48:3543-51.

17. Shen JX, Tama Cl, Hayes RN. Evaluation of automated micro solid phase extraction tips (micro-SPE) for the validation of a LC-MS/MS bioanalytical method. J Chromatogr B Analyt Technol Biomed Life Sci 2006;843: 275-82.

18. Shen JX, Krishna G, Hayes RN. A sensitive liquid chro- matography and mass spectrometry method for the determination of posaconazole in human plasma. $J$ Pharm Biomed Anal 2007;43:228- $\overline{3} 6$.

19. Vogeser M, Fleischer C, Meiser B, Groetzner J, Spöhrer $U$, Seidel D. Quantification of sirolimus by liquid chromatography-tandem mass spectrometry using on-line solid-phase extraction. Clin Chem Lab Med 2002;40: 40-5.

20. Vogeser M, Spöhrer U, Schiel X. Determination of itraconazole and hydroxyitraconazole in plasma by use of liquid chromatography-tandem mass spectrometry with on-line solid-phase extraction. Clin Chem Lab Med 2003; 41:915-20. 